hypotension, and the clip partially detached from the mitral leaflets in three individuals. Following therapy, a sustained reduction in mitral regurgitation was observed in most patients over 6 months.

This study has shown that use of a metal clip device for edge-to-edge percutaneous mitral valve repair is safe and technically feasible, and can also lessen symptoms of mitral regurgitation. Although the authors acknowledge that the technique does not seem to prevent mitral regurgitation in all patients, the promising results have led to further evaluation of its risks and benefits in a phase II randomized trial.

Claire Braybrook

Original article Feldman et al. (2005) Percutaneous mitral valve repair using the edge-to-edge technique; six-months results of the EVEREST phase I clinical trial. J Am Coll Cardiol 46:1799-1809

\section{Pulmonary artery catheter use in advanced heart failure: the ESCAPE trial}

Use of a pulmonary artery catheter (PAC) for hemodynamic monitoring in patients with severe heart failure is controversial, partly because its effect on patients' outcomes is uncertain.

The Evaluation Study of Congestive Heart Failure and Pulmonary Catheterization Effectiveness (ESCAPE) trial was designed to establish whether therapy guided by PAC monitoring and clinical assessment improves outcomes, with fewer days spent in hospital over 6 months, compared with therapy guided by clinical assessment alone. This multicenter, randomized trial was sponsored by the National Heart, Lung, and Blood Institute, and enrolled 433 patients with severe symptomatic heart failure.

Although in-hospital adverse events occurred more frequently among patients who had received therapy guided by $\mathrm{PAC}$, there was no overall increase in mortality, or the length of time spent in hospital. A consistent trend for improved cardiac function and exercise ability was observed in recipients of PAC-guided therapy, and the trial also found that patients with advanced heart failure frequently have a preference for increasing quality, rather than length of life.

In conclusion, there is no indication for routine use of PACs in patients admitted to hospital with heart failure, although this might be a useful approach for patients in whom initial therapy fails to relieve symptoms of clinical congestion. Claire Braybrook

Original article Binanay C et al. (2005) Evaluation study of congestive heart failure and pulmonary artery catheterization effectiveness: the ESCAPE trial. JAMA 295: 1625-1633

\section{Simvastatin as prophylactic treatment for cerebral vasospasm}

Cerebral vasospasm is a major cause of morbidity and mortality following aneurysmal subarachnoid hemorrhage (SAH). A recent randomized clinical trial in the US has investigated the ability of simvastatin to reduce vasospasm after aneurysmal SAH.

In this study, 39 patients who had presented to hospital within 48 hours of an SAH were randomized to simvastatin $80 \mathrm{mg}$ daily $(n=19)$ or placebo $(n=20)$ for 14 days. Daily blood samples were collected for analysis of serum markers of brain injury such as $\mathrm{S} 100 \beta$, and laboratory test results were evaluated weekly for early signs of hepatitis or myositis. The primary endpoint was vasospasm defined by a clinical impression of delayed ischemic neurological deficit not related to rebleed, infection or hydrocephalus, in the presence of one or more confirmatory radiographic tests.

The mean plasma S100 $\beta$ concentration was found to be almost fourfold lower in patients receiving simvastatin than in those receiving placebo $(P<0.01)$, and the mean von Willebrand factor concentration was nearly two fold lower in the treatment arm $(P<0.05)$. Five of the 19 simvastatin patients (26\%) showed evidence of vasospasm development, compared with 12 of the 20 patients (60\%) receiving placebo. A significant decrease was observed in the maximum mean middle cerebral artery transcranial Doppler velocity for the simvastatin group compared with placebo $(P<0.01)$. No patients developed symptoms of hepatitis or myopathy.

The authors conclude that the use of simvastatin as prophylaxis against delayed cerebral ischemia after aneurysmal SAH is safe and well tolerated, and reduces the incidence of vasospasm.

Christine Kyme

Original article Lynch JR et al. (2005) Simvastatin reduces

vasospasm after aneurysmal subarachnoid hemorrhage: results of a pilot randomized clinical trial. Stroke 36: 2024-2026 